\title{
Evaluation of a Screening and Counseling Tool for Alcohol Misuse: A Virginia Practice Support and Research Network (VaPSRN) Trial
}

\author{
Scott M. Strayer, MD, MPH, Sandra L. Pelletier, PhD, Lisa K. Rollins, PhD, \\ Steve W. Heim, MD, MSPH, Karen S. Ingersoll, PhD, Lee M. Ritterband, PhD, \\ and John B. Schorling, $M D, M P H$
}

Background: Surveys reveal limited screening and counseling for alcohol misuse by primary care physicians despite evidence-based recommendations. We developed and evaluated an alcohol screening and misuse counseling tool designed to assist clinicians at the point of care (POC).

Methods: This was a mixed methods, prospective cohort study conducted with licensed clinicians in a practice-based research network. A software tool was designed to guide clinicians through evidencebased alcohol misuse assessment and interventions.

Results: Participants $(\mathrm{N}=12)$ used the tool an average of 3 sessions and $71 \%$ were satisfied with the tool. Participants increased their ability to differentiate between patients who are "at risk" drinkers versus those with alcohol use disorders including dependence/abuse $(21 \% ; t=2.4 ; P=.04)$. Thematic analysis of interviews suggests that barriers to overall use included perceptions of alcohol use; clinical need to intervene; time; and issues with use of technology, most often at the POC. However, the tool added confidence and a valuable framework for interventions and was valued as an educational tool. Users felt that increased training and practice could increase comfort and impact future POC use. Increased POC usability also may be achieved through simplification of the tool and additional flexibility in options for POC use.

Conclusions: A computer-assisted counseling tool for alcohol misuse and abuse can be implemented in primary care settings and shows promise for improving physician screening and interventions for alcohol misuse. To enhance utility in daily clinical practice we recommend design enhancements and strategies to enhance usage as described in this research. ( $\mathrm{J}$ Am Board Fam Med 2012;25:605-613.)

Keywords: Alcohol Drinking, Alcoholism, Behavioral Counseling, Practice-based Research, Practice-based Research Networks

Alcohol consumption is the leading cause of death in the United States among 15 to 45 year olds ${ }^{1}$ and is second only to tobacco use and unhealthy diet/physical inactivity in actual cause of death for all ages. ${ }^{2}$ An estimated 75,000 deaths per year are attributed to alcohol $^{3}$ because of multiple adverse health consequences, including increased risk of violence or injury, cirrhosis of the liver, cancer, and other chronic illnesses, incurring $\$ 185$ billion annually in health care ex-

This article was externally peer reviewed.

Submitted 28 February 2011; revised 22 January 2012; accepted 25 January 2012.

From the Department of Family and Preventive Medicine, University of South Carolina School of Medicine, Columbia (SMS); the Department of Family Medicine (SLP, LKR, SWH), Department of Psychiatry and Neurobehavioral Sciences (KSI, LMR), and Department of Medicine (JBS), University of Virginia Health System, Charlottesville.

Funding: This work was funded by the National Institute on Alcohol Abuse and Alcoholism grant no. 1 R41 AA16030-01 (SMS, primary investigator).

Conflict of interest: none declared.

Corresponding author: Scott M. Strayer, MD, MPH, Department of Family and Preventive Medicine, University of South Carolina School of Medicine, 3209 Colonial Drive, Columbia, SC 29203 (E-mail: sstrayer@uscmed.sc.edu). 
penditures in the United States. ${ }^{2}$ The prevalence estimates for unhealthy alcohol use in primary care settings range from $2 \%$ to $9 \%$ of adults with alcohol dependence to a high of $29 \%$ with "risky use" of alcohol. ${ }^{4}$

Clinically appropriate and effective identification tools and treatment for alcohol problems have been developed. ${ }^{5}$ A US Preventive Services Task Force review concluded that behavioral counseling interventions for risky/harmful alcohol use in primary care could reduce risky/ harmful alcohol use. ${ }^{6}$ Brief interventions that include counseling, especially motivational interviewing approaches, have received empirical support ${ }^{7}$ and expert recommendation. ${ }^{8}$ Current recommendations are that all adults should be screened for unhealthy alcohol use with a validated screening instrument such as the CAGE questionnaire or the Alcohol Use Disorders Identification Test. $^{9}$

Despite these recommendations, clinicians are not using valid identification tools and interventions for unhealthy alcohol use in clinical practice regularly, and usage rates vary widely between locations and settings. ${ }^{10,11}$ A survey of family physicians and internists found that only $64.9 \%$ of respondents screened $80 \%$ to $100 \%$ of their patients for unhealthy alcohol use during the initial visit and a mere $34.4 \%$ screened the same percentage of patients during an annual visit. ${ }^{12}$ Another national survey of primary care physicians reported similar findings: Only $13 \%$ use a formal alcohol screening tool when asking new outpatients about alcohol use. $^{13}$

Despite the proven success of physician-led interventions for alcohol misuse, ambitious efforts to implement primary care alcohol counseling have not succeeded. ${ }^{14,15}$ These problems are caused by a number of barriers, including confusion as to what constitutes alcohol misuse, ${ }^{16}$ fear that asking about drinking could harm the patient-provider relationship, ${ }^{17-19}$ stigmatization of substance abuse, ${ }^{16,20}$ skepticism about the effectiveness of alcohol counseling, ${ }^{16,19,21}$ lack of time, ${ }^{17-19,22}$ inadequate training, ${ }^{16,23,24}$ and a belief that patients will not honestly disclose their drinking practices. ${ }^{18,19,25}$ These barriers may be exacerbated by evidence that moderate levels of drinking ( 1 to 2 drinks per day) have proven beneficial for health. ${ }^{26,27}$
To address this clinical need and physician barriers to alcohol misuse and abuse counseling, we developed a point-of-care (POC) software tool to assist clinicians in providing patienttailored alcohol counseling while facilitating provider adherence to evidence-based practice guidelines. Whereas previous studies have examined electronic reminders, ${ }^{28,29}$ electronic screening tools, ${ }^{30}$ and Internet-based programs for alcohol interventions, ${ }^{31-33}$ our study is the first known investigation to assess a tool to assist primary care physicians with screening and subsequent counseling for alcohol misuse. Study aims were to establish the feasibility and technical merit of the Alcohol Misuse Intervention Tool (AMIT) prototype by conducting formal usability testing of the prototype with clinicians in primary care settings; measuring utilization of the prototype and changes in clinician behavior, knowledge, attitudes, and perceived self-efficacy in applying evidence based counseling strategies before and after the intervention; and qualitative evaluation of physician tool use in primary care settings.

\section{Methods}

\section{Development of the Alcohol Counseling Tool}

The AMIT software tool was designed to assist clinicians in providing patient-tailored alcohol counseling while facilitating adherence to evidence-based practice guidelines. We have previously described our software development process in detail. ${ }^{34,35}$ Briefly, a content development team was assembled and met biweekly for approximately 4 months. The team included 3 experts in behavioral change in addition to the primary investigator (SMS), who led the team. The entire content development team effort was coordinated and directed by the primary investigator (SMS), who has led the development of several clinician behavioral change support tools. A prototype version of the tool was designed as an interactive, web-distributable, clinical decision support software application for computers running either the Palm (Palm, Inc, Sunnyvale, CA) or Microsoft Pocket PC operating systems (Microsoft Corp, Redmond, WA; eg, personal digital assistants [PDAs]). The functionality was later extended during the trial to accommodate use on other computing systems (eg, web-based 
for desktop PC and smartphone use). The tool included algorithms based on the 5 As (ask, advise, assess, assist, and arrange), ${ }^{36}$ the Transtheoretical model of health behavior change, ${ }^{37}$ and motivational interviewing. ${ }^{38}$ Through a pointand-click interface, the AMIT was designed to (1) guide clinicians in screening for alcohol abuse and dependence based on the National Institute on Alcohol Abuse and Alcoholism's guidelines ${ }^{39}$; (2) assist clinicians in delivering a scripted interview to assess the patient's readiness to change; (3) provide a scripted motivational interview tailored to the patient's stage of change; (4) provide stage-relevant tools including risk calculators, drug information and dosing, abridged information about pertinent clinical guidelines, and local and national resources; and (5) track clinician behavior during the patient encounter (ie, identify the clinician using the application, record a time and date stamp each time the application is opened, record which sections of the application are accessed, and time spent in each).

\section{Study Enrollment}

This study was approved by the University of Virginia Health System Institutional Review Board. Clinicians were recruited from practices affiliated with the practice-based research network, which includes 10 academic practices and community-based practices in the University of Virginia Health System. Before the study, clinicians who enrolled provided consent and answered a questionnaire before they participated in a demonstration of features and functionality of the AMIT tool and role played using the tool in a simulated patient encounter with the study coordinator.

\section{Questionnaire Before and After the Study}

We administered a questionnaire to determine clinician knowledge, attitudes, comfort, and behaviors related to alcohol counseling based on previous instruments we have developed ${ }^{35}$ and other previously validated instruments. ${ }^{12,13}$ The questionnaire also included basic information about enrolled clinicians' clinical training and experience. Behavioral theories informing construction of questionnaire items included the 5 As, ${ }^{36}$ stages of change ${ }^{37}$ (based on the Transtheoretical model), and motivational interviewing. ${ }^{38}$ Content experts (SMS, KSI, JBS) reviewed the questionnaire for content validity. The final questionnaire consisted of 34 pre- and postitems, with an additional 4 items designed to measure usability in the questionnaire completed after the study.

\section{Tool Use}

Clinicians were asked to use the tool as they saw fit during the 12-month study period. Prototype usage was monitored during the study. For users of the PDA-based tool, a data usage log was saved on the device and transmitted to a collection database via the Internet when the device was synchronized (synchronization is the process of connecting a handheld device to a desktop computer and updating data on both devices so that the information is the same in both locations). For participants who used the web-based tool, these usage data were collected via web usage logs, for which each clinician had a unique username and password. Collective and individualized activity reports provided information about synchronization, total synchronizations, and average sessions per synchronization. Data on each user's total sessions, average pages viewed per session, unique pages viewed per session, and page content was reviewed. Reviews of synchronizations and usage data were conducted and E-mails offering assistance with technical or other barriers to use were sent to participants who had not synchronized. At approximately 6 to 8 weeks after the web-based tool was introduced, an E-mail reminder was sent to all participants who had limited or no use of the tool. Another E-mail reminder offering technical support and additional practice was sent midway through the study. During the last half of the study, E-mail reminders with tips and articles related to alcohol counseling were sent 2 additional times and a final reminder was sent 2 weeks before the study ended.

\section{Qualitative Assessment of Tool Use}

At the end of the study period, participants completed the poststudy questionnaire and then participated in a 45 - to 60 -minute interview about their use of and satisfaction with the tool. A semistructured interview format was used by the study coordinator (SLP) during one-on-one interviews with participants. Questions addressed 
use of the tool, nature of use, strengths and weaknesses of the tool, barriers to use, and recommended changes. These interviews were audio-recorded and transcribed by the study coordinator. Content analysis and identification of themes was performed by the study coordinator (SLP) and content and themes were reviewed and verified by content experts on the study team (SMS, JBS). Emergent design principles and an inductive approach ${ }^{40}$ were used because of limited data and theories about the use of clinical assessment and intervention tools for alcohol counseling by primary care physicians.

\section{Statistical Analysis}

In addition to qualitative analysis of the structured interview results to identify and report general themes, paired $t$ tests were performed to assess mean differences in the 3 main subscales (physician knowledge about and behaviors and comfort with alcohol screening and intervention practices) from before to after the study. Because this was a pilot study designed a priori to determine feasibility (not efficacy), the sample size was too small to have adequate power to make statistical inferences from the data. Therefore, we examined the results of the $t$ tests to identify trends and generate hypotheses to be tested in a larger, randomized, controlled trial. All quantitative analyses were conducted using SPSS for Windows version 11 (IBM/SPSS, Inc., Chicago, IL).

\section{Results}

Initial recruitment began in February 2008. In May 2008 recruitment was broadened to include clinicians interested in using a web-based tool, and recruitment ended in July 2008. A total of 19 licensed clinicians were enrolled, including 2 family medicine faculty development fellows, 3 residents ( 1 in postgraduate year 3 and 2 in postgraduate year 2), and 1 nurse practitioner. Participants practiced in 3 university-affiliated practices ( 2 urban, 1 rural) and 3 community-based practices. Twelve participants $(63 \%)$ completed the trial based on returning before and after intervention surveys (see Table 1 for demographics). Sixteen participants (84\%) completed a semistructured interview after the trial. Reasons for drop outs included family medical leave $(\mathrm{n}=$ 1), loss of preintervention survey by participant
Table 1. Participant Demographics

\begin{tabular}{lr}
\hline Clinician type/affiliation & \\
Faculty physician & 6 \\
Community physician & 1 \\
Fellow physician & 2 \\
Resident physician & 1 \\
PGY 3 & 1 \\
PGY 2 & 1 \\
Nurse practitioner & 58 \\
Male sex (\%) & 42 \\
Age (mean years) & 8 \\
Practice type & 1 \\
Academic & 2 \\
Academic internal medicine & 1 \\
Academic-rural & \\
Community-urban & \\
\hline
\end{tabular}

Values provided as $\mathrm{n}$ unless otherwise indicated. PGY, postgraduate year.

$(\mathrm{n}=1)$, and noncompletion of survey after intervention $(\mathrm{n}=5)$. The drop outs included 1 resident, 3 academic faculty, and 3 community physicians ( 4 men, 3 women). Communication at the end of the study indicated reluctance to participate in follow-up because of limited use of the tool and the time lag since the start of the trial.

\section{Use of Tool}

Clinicians had access to the tool for an average of 8.5 months (range, 7-12 months). Technical issues related to PDA use as well as recruitment issues led to adapting the tool in May 2008 so it could be accessed on any web-enabled computing device. Some physicians had difficulties installing the PDA software and synchronizing data and experienced PDA crashes resulting in data loss. In addition, desktop computers were made available in every patient examination room, making a web-enabled version more attractive to some users. Ten of the participants had access to the web-based version of the tool and 2 participants had access to both versions. Details of participant tool use are fully described in Table 2 .

\section{Knowledge, Comfort, and Behaviors Pre- and Post-Intervention}

No statistically significant mean differences were found for physician knowledge (mean increase, $0.70 ; P=.35$ ), physician comfort (mean increase, $0.09 ; P=.93)$, and physician counseling behav- 
Table 2. Alcohol Misuse Intervention Tool (AMIT) Use Data

\begin{tabular}{lc}
\hline Clinicians completing study $(\mathrm{n}=12)$ & \\
Total sessions with AMIT $(\mathrm{n})$ & 27 \\
Use of patient interview section & $19(70)$ \\
$\quad$ (n [\%]) & \\
Use of educational content (n [\%]) & $8(30)$ \\
Sessions per user (mean [range]) & $3(0-10)$ \\
Pages per session (mean) & $16(3-37)$ \\
Session length (includes suspected & $4: 51(10$ sec to 16 min) \\
training), minutes (mean & \\
$\quad$ [range]) & \\
Clinicians using tool* & \\
Total sessions with AMIT & 31 \\
Sessions per user (mean [range]) & $3(1-10)$ \\
Pages per session (mean) & 15 \\
Session length (excludes suspected & $3: 28$ \\
$\quad$ training), minutes & \\
\hline
\end{tabular}

*Includes participants who did not complete study $(\mathrm{n}=11)$.

iors (mean increase, $0.25 ; P=.96$ ). We were only able to analyze 11 completed before and after surveys because one survey before the study was lost by the participant. However, there was an increase in physicians distinguishing between patients who are "at risk" drinkers versus those with alcohol use disorders (eg, dependence/abuse) $(21 \% ; t=2.4 ; P=.04)$.

\section{Qualitative Assessment of Tool Use and Satisfaction}

Semi-structured interviews were conducted with 16 of the enrolled clinicians including 9 of those analyzed in the before and after analysis. We were able to analyze 12 interviews (4 were unanalyzed because of technical problems with the audio recording) with 5 faculty physicians, 2 community-based physicians, 2 fellows, 2 residents, and 1 nurse practitioner. Of these 12 participants, 7 had used the tool at least once during the trial, 2 only used it during training, and we did not have use data for 3 of the participants because of technical issues with PDA use logs.

Overall, participants were satisfied with the tool $(71 \%)$ and all participants stated they would use the tool if it was modified. Thematic analysis of interviews suggested that barriers to overall use included perceptions of alcohol use; clinical need to intervene; time; and issues with use of technology generally at the POC. However, the tool added confidence and a valuable framework for interventions and was valued as an educational tool. Users felt that increased training and practice could increase comfort and impact future POC use. Increased POC usability also may be achieved through tool simplification and additional flexibility in POC use options suggested by participants (see Table 3 ).

\section{Discussion}

We developed a computer-assisted screening and counseling tool for alcohol misuse and studied its use and uptake in primary care settings. Our study uncovered important barriers to use and suggestions for improvement that are necessary to achieve more widespread use and clinical utility of this POC tool in primary care settings. When clinicians used the tool, the frequency, length of time, and page views were consistent with brief counseling sessions in primary care, as was using it as an educational reference. The majority of study participants were satisfied with

Table 3. Summary of Suggestions for Tool Modifications and Barriers to Use from Qualitative Analysis

- More patient resources (eg, tailored patient handouts, a web portal for patients)

- Options for targeted use (eg, access to drug dosing in 2 steps, identifying resources)

- Enhancing the tool design for interactivity with patients

- Integrating the tool with electronic medical records

- Completing screening before the physician visit (eg, by patients or other clinical team members)

- Improved navigation through the tool so users can see where they are going in the interview

- Providing assistance with billing and reimbursement
- Perceptions that few patients have alcohol misuse/abuse problems

- Multiple competing demands in primary care settings

- Lack of time

- Issues with technology at the point of care 
the tool and all participants stated that they would use this tool in practice if it was modified based on the feedback from the study. Suggestions for tool modifications and barriers to use are described in Table 3.

Although most of these barriers have been previously cited, we were struck by the dissonance physicians seemed to exhibit regarding perceived prevalence of alcohol misuse and abuse among their patients as contrasted with epidemiologic data supporting the high death toll ${ }^{2}$ and high prevalence of risky alcohol use among the general population. ${ }^{8}$ We did not find this barrier previously reported in our literature review and would recommend exploring physicians' perceptions fully as a part of interventions aimed at increasing screening and counseling for alcohol use and misuse. Based on their perceptions, there may be a need for further education and training about the prevalence and public health impacts of unsafe alcohol use.

Additional training and practice with the tool also were identified as important components of successful implementation. Finally, providing resources that add value to using the tool (see Table 3) may be important for successful implementation. These are components and approaches that are being integrated into similar behavioral counseling tools for smoking cessation and diet/exercise that we are currently developing, ${ }^{41,42}$ and their effect on implementation will be evaluated.

There are several limitations of this study that should be considered when interpreting these results. Only a small percentage of practice-based research network clinicians (approximately 9\%) enrolled in the pilot trial to test the tool, potentially limiting the generalizability of our findings. In addition, approximately one third of participants $(37 \%)$ dropped out of the study, also limiting generalizability and introducing bias. Another potential source of bias may have been introduced by having study team members conduct the semistructured interviews after the intervention. We did not find statistically significant differences between physician assessment before and after the intervention, but the study was not powered to see small differences in study measures and could represent type II error. Finally, because of multiple comparisons in our analysis, there is the possibility of a type I error in our statistically significant finding that physicians were more likely to report assessing for "at risk" drinking versus dependence/abuse. This was a limitation of the study from the outset and underscores that it was designed as a pilot to generate hypotheses and identify trends. Nevertheless, finding at least one change in physician behavior may be important and is consistent with our previously tested tools. In a previous study, we developed a modular lifestyle intervention tool to address smoking cessation and unhealthy weight and found that physicians were more likely to advise patients to stop smoking and were more likely to arrange follow-up for overweight patients who wanted to lose weight. In addition, the tool increased the overall use of the 5 As during patient encounters and increased general counseling behaviors for both smoking cessation and weight loss. ${ }^{43} \mathrm{We}$ recommend further evaluation of the AMIT tool with suggested improvements in a larger sample to definitively explore the efficacy of this approach with alcohol use/misuse.

Computer-assisted behavioral change counseling has the potential to enhance interventions in primary care settings and can assist physicians with tailoring interventions for individual patients. Results from several studies indicate that providing people with unhealthy behaviors with a personalized report based on patient readiness to change enhances cessation rates. ${ }^{44-50} \mathrm{~A}$ Cochrane review reported a meta-analysis of 17 trials using materials tailored to the characteristics of individual patients. Although part of the effect could be attributable to the additional contact or assessment required to obtain individual data, this systematic review found that tailored materials increase rates of addiction management rates over and above standard materials and untailored materials (OR, 1.42; 95\% CI, 1.261.61). ${ }^{51}$ Implementing this approach using technologies such as smartphones and integration with electronic medical records has the potential to increase the reach of the intervention because of rapidly increasing physician adoption of these technologies at the POC.

\section{Conclusions}

This pilot study illustrates challenges and potential solutions for implementing a computerassisted counseling tool for alcohol misuse and 
abuse in primary care settings. Based on our experience with implementation, we recommend evaluating integration of this tool within electronic medical records, where it can easily be made available during patient encounters and documentation can be accomplished automatically during the interview. We also recommend evaluating this approach with other medical staff and ancillary providers to determine if having other staff using this approach can augment or complement physician interventions. For example, other health care staff might complete a basic assessment and intervention with patients and have a tailored report that physicians could use to make key counseling points during an office visit. Our next steps are to conduct additional design enhancements and usability testing of the tool and test the tool in a randomized, controlled trial with sufficient power to determine differences in patient outcomes.

\section{References}

1. McGinnis JM, Foege WH. Actual causes of death in the united states. JAMA. 1993;270:2207-12.

2. Mokdad AH, Marks JS, Stroup DF, Gerberding JL. Actual causes of death in the united states, 2000. JAMA 2004;291:1238-45.

3. Centers for Disease Control and Prevention (CDC). Alcohol-attributable deaths and years of potential life lost-United States, 2001. MMWR Morb Mortal Wkly Rep 2004;53:866-70.

4. Reid MC, Fiellin DA, O'Connor PG. Hazardous and harmful alcohol consumption in primary care. Arch Intern Med 1999;159:1681-9.

5. National Institute on Alcohol Abuse and Alcoholism. The tenth special report to the U.S. Congress on alcohol and health. 2000. NIH publication no. 00-1583. Rockville, MD: National Institutes of Health; :429-443.

6. Whitlock EP, Green CA, Polen MR, et al. Behavioral counseling interventions in primary care to reduce risky/harmful alcohol use: a summary of the evidence for the U.S. Preventive Services Task Force. Ann Intern Med 2004;140:557-68.

7. Rubak S, Sandbaek A, Lauritzen T, Christensen B. Motivational interviewing: a systematic review and meta-analysis. Br J Gen Pract 2005;5 5:305-12.

8. Saitz R. Clinical practice. Unhealthy alcohol use. N Engl J Med 2005;352:596-607.

9. Fiellin DA, Reid MC, O'Connor PG. Screening for alcohol problems in primary care: a systematic review. Arch Intern Med 2000;160:1977-89.
10. Institute of Medicine. Broadening the base of treatment for alcohol problems. Washington, DC: National Academies Press; 1990.

11. National Institute on Alcohol Abuse and Alcoholism (NIAAA). Ninth special report to the U.S. Congress on alcohol and health from the secretary of health and human services. NIH publication no. 97-4017. Rockville, MD: National Institutes of Health; 1997.

12. Spandorfer JM, Israel Y, Turner BJ. Primary care physicians' views on screening and management of alcohol abuse: Inconsistencies with national guidelines. J Fam Pract 1999;48:899-902.

13. Friedmann PD, McCullough D, Chin MH, Saitz R. Screening and intervention for alcohol problems. A national survey of primary care physicians and psychiatrists. J Gen Intern Med 2000;15: 84-91.

14. Babor TE, Higgins-Biddle J, Dauser D, Higgins $\mathrm{P}$, Burleson JA. Alcohol screening and brief intervention in primary care settings: Implementation models and predictors. J Stud Alcohol 2005;66: 361-8.

15. Funk M, Wutzke S, Kaner E, et al. A multicountry controlled trial of strategies to promote dissemination and implementation of brief alcohol intervention in primary health care: findings of a World Health Organization collaborative study. J Stud Alcohol 2005;66:379-88.

16. Rush B, Ellis K, Crowe T, Powell L. How general practitioners view alcohol use. Clearing up the confusion. Can Fam Physician 1994;40:1570-9.

17. Arborelius E, Damstrom Thakker K. Why is it so difficult for general practitioners to discuss alcohol with patients? Fam Pract 1995;12:419-22.

18. Lock CA, Kaner E, Lamont S, Bond S. A qualitative study of nurses' attitudes and practices regarding brief alcohol intervention in primary health care. J Adv Nurs 2002;39:333-42.

19. Beich A, Gannik D, Malterud K. Screening and brief intervention for excessive alcohol use: qualitative interview study of the experiences of general practitioners. BMJ 2002;325:870.

20. Bander KW, Goldman DS, Schwartz MA, Rabinowitz E, English JT. Survey of attitudes among three specialties in a teaching hospital toward alcoholics. J Med Educ 1987;62:17-24.

21. Aira M, Kauhanen J, Larivaara P, Rautio P. Differences in brief interventions on excessive drinking and smoking by primary care physicians: qualitative study. Prev Med 2004;38:473-8.

22. Anderson P, Kaner E, Wutzke S, et al. Attitudes and managing alcohol problems in general practice: an interaction analysis based on findings from a WHO collaborative study. Alcohol Alcohol 2004;39:351-6. 
23. Cornuz J, Zwahlen S, Jungi WF, et al. A vaccine against nicotine for smoking cessation: a randomized controlled trial. PLoS One 2008;3:e2547.

24. Cornuz J, Ghali WA, Di Carlantonio D, Pecoud A, Paccaud F. Physicians' attitudes towards prevention: Importance of intervention-specific barriers and physicians' health habits. Fam Pract 2000;17:535-40.

25. Thom B, Tellez C. A difficult business: detecting and managing alcohol problems in general practice. Br J Addict 1986;81:405-18.

26. Thun MJ, Peto R, Lopez AD, et al. Alcohol consumption and mortality among middle-aged and elderly U.S. adults. N Engl J Med 1997;337: 1705-14.

27. National Institute on Alcohol Abuse and Alcoholism (NIAAA). State of the science report on the effects of moderate drinking. Rockville, MD: National Institutes of Health, Department of Health and Human Services; 2003. Available from http:// pubs.niaaa.nih.gov/publications/ModerateDrinking-03. htm. Accessed July 15, 2012.

28. Williams EC, Lapham G, Achtmeyer CE, Volpp B, Kivlahan DR, Bradley KA. Use of an electronic clinical reminder for brief alcohol counseling is associated with resolution of unhealthy alcohol use at follow-up screening. J Gen Intern Med 2010; 25(Suppl 1):11-7.

29. Williams EC, Achtmeyer CE, Kivlahan DR, et al. Evaluation of an electronic clinical reminder to facilitate brief alcohol-counseling interventions in primary care. J Stud Alcohol Drugs 2010;71: $720-5$.

30. Farrell SP, Mahone IH, Zerull LM, et al. Electronic screening for mental health in rural primary care: implementation. Issues Ment Health Nurs 2009;30:165-73.

31. Saitz R, Palfai TP, Freedner N, et al. Screening and brief intervention online for college students: the ihealth study. Alcohol Alcohol 2007;42:28-36.

32. Kypri K, Saunders JB, Gallagher SJ. Acceptability of various brief intervention approaches for hazardous drinking among university students. Alcohol Alcohol 2003;38:626-8.

33. Sinadinovic K, Berman AH, Hasson D, Wennberg $\mathrm{P}$. Internet-based assessment and self-monitoring of problematic alcohol and drug use. Addict Behav 2010;35:464-70.

34. Heim SW, Nadkarni M, Rollins LK, et al. Modular lifestyle intervention tool: a handheld tool to assist clinicians in providing patient-tailored counseling. Ann Fam Med 2005;3(Suppl 2):S65-7.

35. Strayer SM, Rollins LK, Martindale JR. A handheld computer smoking intervention tool and its effects on physician smoking cessation counseling. J Am Board Fam Med 2006;19:350-7.

36. Whitlock EP, Orleans CT, Pender N, Allan J. Evaluating primary care behavioral counseling in- terventions: an evidence-based approach. Am J Prev Med 2002;22:267-84.

37. Prochaska JO, DiClemente CC. Stages and processes of self-change of smoking: toward an integrative model of change. J Consult Clin Psychol 1983;51:390-5.

38. Miller WR, Rollnick S. Motivational interviewing: preparing people for change. Vol 2. New York: Guilford Press; 2002.

39. National Institute on Alcohol Abuse and Alcoholism. Helping patients who drink too much: a clinician's guide. NIH publication no. 07-3769. Rockville, MD: National Institutes of Health; 2005.

40. Strauss A, Corbin J. Basics of qualitative research: techniques and procedures for developing grounded theory. 3rd ed. Thousand Oaks, CA: Sage Publications; 1998.

41. Strayer SM, Pelletier SL, Rollins LK, et al. Qualitative evaluation and usability of a web-based smoking cessation counseling tool based on motivational interviewing. Fam Med 2011;43(Suppl 1). Available at: http://www.stfm.org/fmsup/napcrg/ fmconferencesupplement.cfm? confid $=135$. Accessed July 30, 2012.

42. Strayer SM, Pelletier SL, Rollins LK, et al. Design and usability of a web-based weight loss counseling tool based on motivational interviewing. Family Medicine. 2011;43(Suppl 1). Available at: http://www. stfm.org/fmsup/napcrg/fmconferencesupplement.cfm? confid=135. Accessed July 30, 2012.

43. Heim SW, Strayer SM, Nadkarni M, et al. Improving physician health behavior counseling. Fam Med 2006;38(Suppl 1). Available at: http://www.stfm.org/ fmsup/napcrg/fmconferencesupplement.cfm? confid= 129. Accessed July 30, 2012

44. Prochaska JO, Velicer WF, Redding C, et al. Stage-based expert systems to guide a population of primary care patients to quit smoking, eat healthier, prevent skin cancer, and receive regular mammograms. Prev Med 2005;41:406-16.

45. Prochaska JO, Velicer WF, Fava JL, Rossi JS, Tsoh JY. Evaluating a population-based recruitment approach and a stage-based expert system intervention for smoking cessation Addict Behav 2001;26:583-602.

46. Strecher VJ, Shiffman S, West R. Moderators and mediators of a web-based computer-tailored smoking cessation program among nicotine patch users. Nicotine Tobacco Res 2006;8(Suppl 1):S95-101.

47. Strecher VJ, Shiffman S, West R. Randomized controlled trial of a web-based computer-tailored smoking cessation program as a supplement to nicotine patch therapy. Addiction 2005;100:682-8.

48. Strecher VJ, Marcus A, Bishop K, et al. A randomized controlled trial of multiple tailored messages for smoking cessation among callers to the cancer information service. J Health Commun 2005; 10(Suppl 1):105-18. 
49. Strecher VJ, Kreuter M, Den Boer DJ, et al. The effects of computer-tailored smoking cessation messages in family practice settings. J Fam Pract 1994;39:262-8.

50. Dijkstra A, De Vries H, Roijackers J, et al. Tailored interventions to communicate stage- matched information to smokers in different motivational stages. J Consult Clin Psychol 1998;66: 549-57.

51. Lancaster T, Stead LF. Self-help interventions for smoking cessation. Cochrane Database Syst Rev 2005;(3):CD001118. 